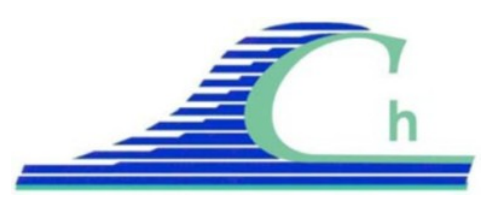

XII ${ }^{\text {èmes }}$ Journées Nationales Génie Côtier - Génie Civil

Cherbourg, 12-14 juin 2012

DOI:10.5150/jngcgc.2012.029-F＠ Editions Paralia CFL

disponible en ligne - http://www.paralia.fr - available online

\title{
Dynamique spatio-temporelle du matériel végétal grossier dans l'estuaire de la Gironde
}

\author{
Ana FUENTES CID ${ }^{1}$, Henri ETCHEBER ${ }^{1}$, Sabine SCHMIDT ${ }^{1}$, \\ Aldo SOTTOLICHIO ${ }^{1}$, Eric DE OLIVEIRA ${ }^{2}$, \\ Georges OGGIAN ${ }^{1}$, Hervé DERRIENIC ${ }^{1}$
}

\author{
1. Univ. Bordeaux 1, UMR/CNRS 5805 EPOC, Avenue des facultés, 33405, \\ Talence CEDEX, France. \\ a.fuentes@epoc.u-bordeaux1.fr \\ 2. LNHE, EDF R\&D, Chatou. France.
}

\section{Résumé :}

Les débris végétaux provenant des bassins versants peuvent transiter vers les estuaires et ainsi contribuer au matériel présent dans ces systèmes. Or les études visant à comprendre le fonctionnement d'un estuaire macrotidal et très turbide, comme celui de la Gironde, sont en général ciblées sur les sédiments fins cohésifs et en particulier sur leur évolution spatio-temporelle. Ainsi, jusqu'à présent, les études n'ont pas, ou rarement, tenu compte de la fraction grossière $(>315 \mu \mathrm{m})$ du matériel allochtone, qui joue son propre rôle dans le milieu et suit une dynamique qui reste encore à élucider.

En effet, bien que peu abondante, la présence de ce matériel peut être une source de nuisances pour les activités impliquant la filtration de grands volumes (pêche, CNPE). Ce travail vise donc à mieux comprendre la dynamique saisonnière de la fraction grossière qui transite dans l'estuaire de la Gironde, un milieu extrêmement turbide, où la charge en matières en suspension peut dépasser plusieurs grammes par litre.

Depuis l'été 2010, un suivi mensuel a été mis en place afin d'évaluer la quantité et la nature du matériel grossier $(>315 \mu \mathrm{m})$ présent dans l'estuaire amont et central de la Gironde. En parallèle, a été conduite une analyse des conditions hydrologiques et de la base de données qui enregistre le fonctionnement du circuit de refroidissement $\mathrm{du}$ Centre Nucléaire Production Electrique (CNPE) du Blayais.

Ceci a permis de montrer un lien entre la présence marquée de débris végétaux grossiers et les crues des tributaires (Garonne, Dordogne) de la Gironde. Le suivi mensuel révèle aussi l'influence de la marée (coefficient de marée, cycle) sur le transport et la présence de ces débris. Cette étude démontre que la fraction grossière $(>315 \mu \mathrm{m})$ présente un comportement hydrodynamique influencé par les mêmes facteurs que celui des sédiments fins, mais avec son cycle propre.

\section{Mots-clés :}

Estuaire de la Gironde - Débris végétaux grossier - Dynamique sédimentaire - CNPE du Blayais 


\title{
Thème 2 - Dynamique sédimentaire
}

\begin{abstract}
:
Plant debris from the watershed can flow into estuaries and contribute to the material in these systems. Up to now, studies about macrotidal and highly-turbid estuaries, like the Gironde, are generally targeted on cohesive sediments, and in particular on their spatiotemporal evolution. So far, studies have not, or rarely, considered the coarse fraction $(>315 \mu \mathrm{m})$ of allochthonous material, which is likely to play its own role in these systems and to follow a dynamic that remains to be elucidated.

Indeed, although scarce, the presence of this material may be a source of nuisance for activities involving the filtration of large volumes (fishing, Nuclear Power Plant (NPP)). This work aims to better understand the seasonal dynamics of the coarse fraction that passes in the Gironde estuary, a highly turbid environment, where the load of fine particles can exceed several grams per liter.

Since summer 2010, a monthly monitoring has been set up to assess the amount and nature of the coarse material $(>315 \mu \mathrm{m})$ present in the central and upper estuary of the Gironde. In parallel, the database of hydrological conditions and of the functioning of the cooling system of NPP-Blayais was analyzed.

This work highlights a strong link between the presence of coarse plant debris and flood of the tributaries (Garonne, Dordogne) of the Gironde. Monthly monitoring also reveals the influence of the tide (tidal coefficient, cycle) on the transport and the presence of debris. This study demonstrates that the coarse fraction $(>315 \mu \mathrm{m})$ has a hydrodynamic behavior influenced by the same factors as that of fine sediments, but with its own cycle.
\end{abstract}

\section{Keywords:}

Estuary of Gironde, Coarse plant debris, Sediment dynamics, Nuclear Power Plant (NPP) of Blayais

\section{Introduction}

L'estuaire de la Gironde apporte en moyenne $60 \%$ du flux total des matières en suspension arrivant au Golfe de Gascogne (JOUANNEAU et al., 1999). Toutefois, cet ordre de grandeur est indicatif, car il existe de grandes incertitudes sur le stock intraestuarien et les flux particulaires effectivement expulsés, et sur leurs variabilités saisonnières et interannuelles. Par ailleurs les débris végétaux provenant du bassin versant peuvent transiter vers l'estuaire et ainsi contribuer au matériel présent dans ce système. Or les travaux visant à comprendre le fonctionnement d'un estuaire macrotidal et très turbide, comme celui-ci de la Gironde, sont en général ciblées sur les sédiments fins cohésifs et en particulier sur leur évolution spatio-temporelle. Aucune étude n'a caractérisé la contribution de la matière grossière d'origine allochtone $(>315 \mu \mathrm{m})$ dans l'estuaire amont et moyen, même si des fortes connexions fluvio-estuariennes sont bien connues lors des crues hivernales. 


\section{XII ${ }^{\text {èmes }}$ Journées Nationales Génie Côtier - Génie Civil \\ Cherbourg, 12-14 juin 2012}

De plus, du fait du changement climatique et des perturbations des débits fluviaux du système Gironde, il y a modification durable des zones d'envasement, déplacées vers la partie fluviale où les sections sont plus réduites et donc plus sensibles au dépôt sédimentaire. Ces évolutions récentes observées en Gironde sont donc susceptibles d'affecter la dynamique du matériel grossier, causant des nuisances pour les activités humaines (pêche, activité industrielle). Ce travail a ainsi pour objectif de mieux connaître la contribution de ce matériel grossier dans l'estuaire et sa dynamique saisonnière en fonction du régime hydrologique du continuum fluvio-estuarien de la Gironde.

\section{Matériels et méthodes}

\subsection{Présentation du site d'étude}

L'estuaire de la Gironde est le plus vaste estuaire d'Europe de l'Ouest avec une surface de $635 \mathrm{~km}^{2}$ et un bassin versant de $71000 \mathrm{~km}^{2}$. Il est situé dans le sud-ouest de la France (figure 1) et ses deux affluents principaux sont la Garonne et la Dordogne.

Cet estuaire est caractérisé par une faible profondeur $(7-10 \mathrm{~m})$, un régime macrotidal $(1,7-5,1 \mathrm{~m})$ et une zone de turbidité élevée, jusqu'à plus de $1 \mathrm{~g} \mathrm{l}^{-1}$ en surface (SOTTOLICHIO et al., 2011). Le régime hydrologique du système Garonne+Dordogne est marqué par des variations saisonnières importantes, une diminution significative des débits au cours des 30 dernières années $\left(1030 \mathrm{~m} . \mathrm{s}^{-1}\right.$ en début des années 80 à $740 \mathrm{~m}^{3} \mathrm{~s}^{-1}$ en fin des années 2000 ; GPMB, Grand Port Maritime de Bordeaux) et des étiages plus prononcés. Les conséquences sont une salinisation du système (DAVID et al., 2011) et une remontée progressive du bouchon vaseux vers les sections fluviales (SOTTOLICHIO et al., 2011).

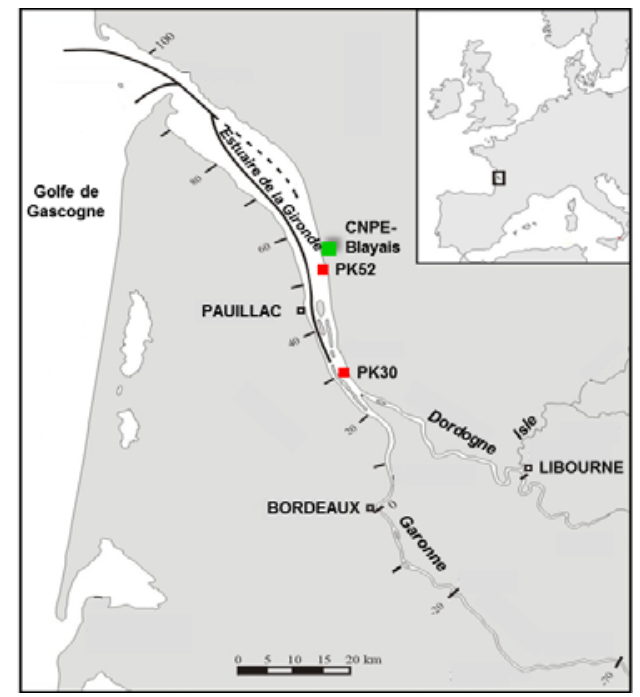

Figure 1. Estuaire de la Gironde et position des sites d'étude. 


\subsection{Prélèvements mensuels et tamisage}

Un suivi mensuel a été réalisé depuis juin 2010 aux sites PK30 et PK52. Outre une filtration de petits volumes d'eau pour déterminer la charge en matières en suspension, des traits de filet (WP-2) ont été effectués à mi-jusant en surface et au fond pour filtrer les grands volumes d'eau nécessaires à la récupération de la fraction grossière. Ces collectes ont été ensuite tamisées pour récupérer le matériel selon deux fractions ( $315 \mu \mathrm{m}-1 \mathrm{~mm} ;>1 \mathrm{~mm})$ et séchées pour en déterminer le poids sec.

\subsection{Campagnes d'échantillonnage au CNPE du Blayais}

Deux campagnes en janvier et février 2011 ont été réalisées dans les eaux de nettoyage du circuit de refroidissement du CNPE du Blayais. La fraction grossière a été récupérée avec un filet (maille : $3 \mathrm{~mm}$ ) (figure 2):

a) au fil d'une marée : quatre prélèvements (pleine mer, mi-jusant, basse mer, et miflot);

b) au fil d'un cycle de marées de 8 jours (un prélèvement par jour, une heure après le mi-jusant).

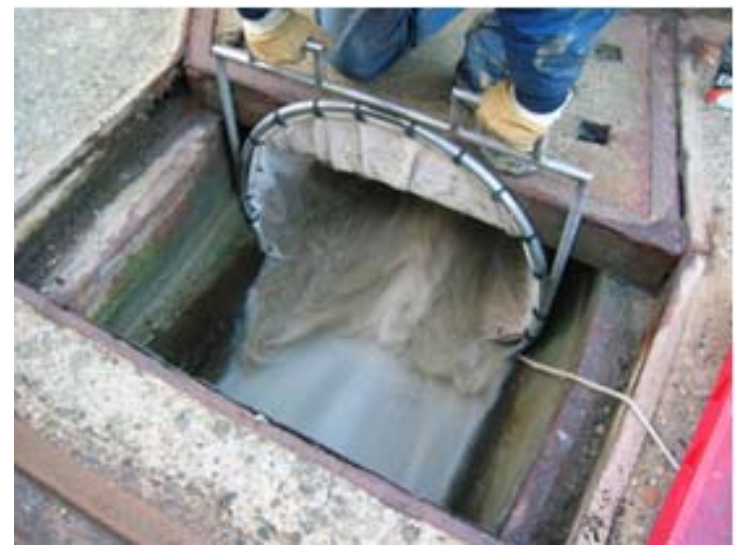

Figure 2. Prélèvement dans les eaux de nettoyage du circuit de refroidissement du CNPE du Blayais.

\section{Résultats}

La figure 3 présente l'évolution des concentrations de matériel grossier (315-1 mm; $>1 \mathrm{~mm}$ ) et des débits (Garonne - Dordogne) au cours de 2011. L'échantillonnage a toujours été réalisé à mi-jusant. En effet, comme le montrent les enregistrements haute fréquence du réseau MAGEST (ETCHEBER et al., 2011), la turbidité en Gironde présente des cycles d'érosion-déposition, typiques des estuaires macrotidaux (ALLEN et al., 1980 ; SOTTOLICHIO \& CASTAING, 1999); lors d'un cycle de marée, les maxima de turbidité sont le plus souvent observés à mi- jusant. 


\section{XII ${ }^{\text {èmes }}$ Journées Nationales Génie Côtier - Génie Civil \\ Cherbourg, 12-14 juin 2012}

Les échantillons ainsi récoltés sont toujours des végétaux d'origine "terrestre" (absence d'espèces aquatiques), en majorité des feuilles de graminées et d'arbres en état de dégradation variable, et dans une moindre mesure des tiges, des herbes ou des racines.

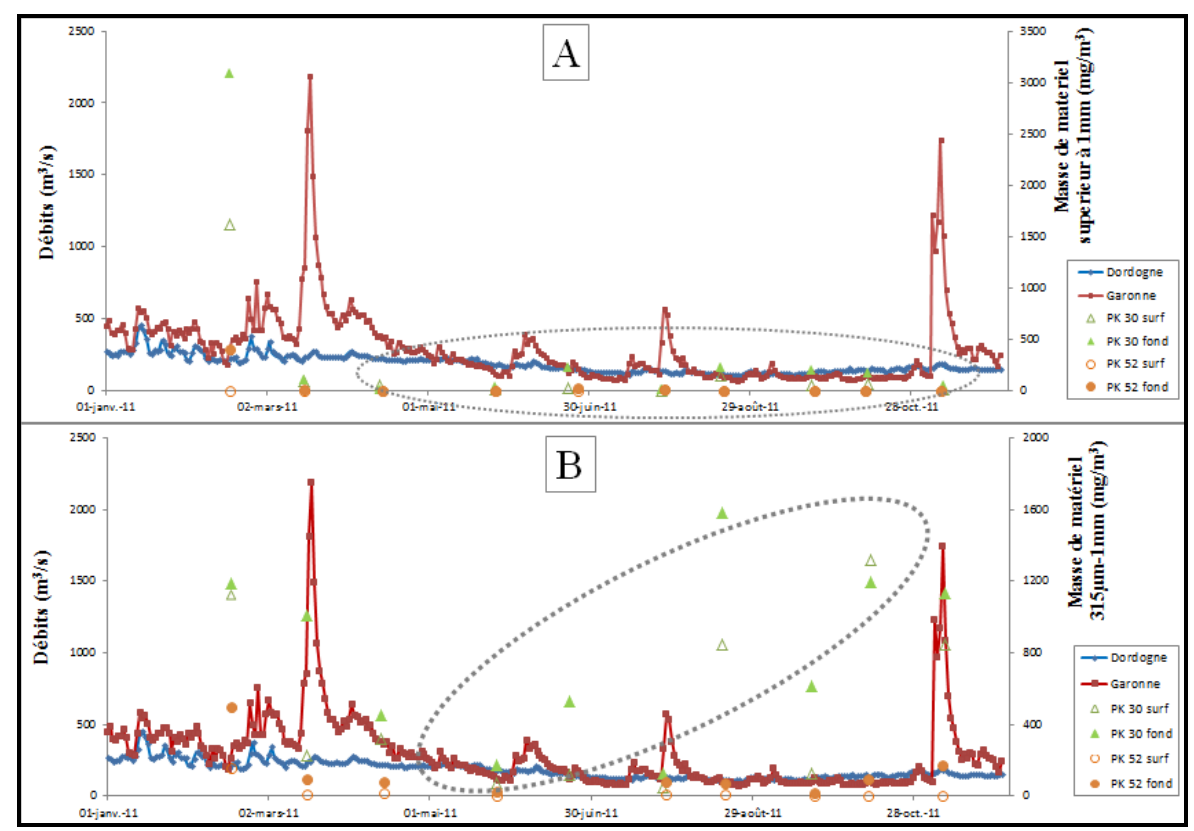

Figure 3. Suivi mensuel (2011) des débits fluviaux et des quantités de matériel grossier (A : >1 mm ; B : 315-1000 $\mu \mathrm{m}$ ) aux sites PK30 et P K 52 de l'estuaire de la Gironde.

Plusieurs observations ressortent de la figure 3 :

a) il y a toujours présence de matériel grossier allochtone dans l'estuaire central, de quelques $\mathrm{mg}$ à plusieurs centaines de $\mathrm{mg} \mathrm{m}^{-3}$ selon les saisons; il est à noter que cela représente en masse environ $0,2 \%$ des matières en suspension, compte-tenu des turbidités des eaux de l'estuaire de la Gironde ;

b) la quantité de matériel grossier est toujours supérieure au fond par rapport à celle de surface ;

c) la fraction $315 \mu \mathrm{m}-1 \mathrm{~mm}$ est toujours plus abondante que celle $>1 \mathrm{~mm}$;

d) au PK 30, il y a toujours plus de matériel récolté qu'au PK 52 ;

e) les deux pics maximum de quantité récoltée correspondent au mois de février et d'octobre 2011 ; les autres mois affichent des valeurs réduites.

Ces résultats prouvent qu'il y a bien une évolution spatio-temporelle des quantités de matière grossière dans l'estuaire de la Gironde, à l'instar du matériel fin. Certaines tendances sont d'ailleurs identiques, comme des concentrations plus fortes en profondeur. De même, les deux campagnes de prélèvement au CNPE du Blayais montrent que les flux de matière lors d'un cycle de marée sont plus faibles lors des étales. Nous avons aussi observé une évolution de la fraction grossière, certes en quantité mais surtout en nature, lors d'un cycle lunaire en lien avec les coefficients de 


\section{Thème 2 - Dynamique sédimentaire}

marée. En effet, alors que des petits morceaux de bois, faciles à remobiliser, dominent lors des coefficients de marée faibles, les débris de feuilles (graminées et arbres) en décomposition sont abondants lors des coefficients de marée élevés. Ceci indique qu'il y a une influence des cycles de marée et lunaire dans le transport et la remise en suspension des débris végétaux.

\section{Discussion}

4.1 Evolution saisonnière de l'importance des fractions grossières dans l'estuaire central Par contre, le lien entre quantités de fraction grossière et débits n'est pas trivial. En effet, eu égard aux apports de débris végétaux depuis le bassin versant vers l'estuaire amont, une corrélation simple avec les débits fluviaux était attendue. Cependant, cette étude met en évidence :

a) un décalage temporel entre les évènements de crues et la présence de matériel végétal grossier dans l'estuaire (qui sera discuté au paragraphe 4.2);

b) deux périodes bien marquées au cours de l'année:

la première, jusqu'en avril, reflète une réponse directe du matériel grossier (les deux fractions) en fonction des pluies hivernales; la seconde, à partir d'avril, quand les apports des bassins versants sont négligeables, avec une augmentation progressive au fil des mois de la fraction $315 \mu \mathrm{m}-1 \mathrm{~mm}$ au PK 30, alors que la fraction $>1 \mathrm{~mm}$ reste faible et assez constante.

Avec la diminution des débits, ceci semble indiquer que les apports de débris végétaux fluviaux peuvent être "bloqués" à l'entrée de l'estuaire. La présence de vase doit ralentir le transport du matériel $(>1 \mathrm{~mm})$ vers l'aval; mais au fil du temps, ce matériel se fractionne (dégradation mécanique très forte, due au mouvement de la marée) et crée des débris plus petits pouvant être déplacés vers l'aval.

\subsection{Impact des crues sur le transport dans l'axe de l'estuaire}

Une fois arrivés dans l'estuaire amont, les débris végétaux connaissent des cycles de dépôts et remises en suspension, similaires à ceux des matières en suspension et un transfert progressif vers l'aval. Afin de chiffrer le décalage de temps entre les apports amont de matériel grossier et sa présence dans la partie centrale, nous avons utilisé les données de débits et les enregistrements du fonctionnement du circuit de refroidissement du CNPE du Blayais. En effet, cette centrale filtre continuellement de grands volumes d'eau estuarienne $\left(160 \mathrm{~m}^{3} / \mathrm{s}\right)$ répartis sur huit pompes. Depuis sa mise en service en 1981, trois épisodes exceptionnels d'arrivées massives de débris végétaux, ayant entraîné des pertes de production (arrêt d'un ou deux réacteurs suite au colmatage des organes de filtration) ont eu lieu en 1996, 2003 et 2009. Même si la présence de matériel a pu être amplifiée par des conditions locales (vent, coefficients de marée, ...), il existe une relation claire entre le décalage temporel du colmatage des tambours (par 


\section{XII ${ }^{\text {èmes }}$ Journées Nationales Génie Côtier - Génie Civil \\ Cherbourg, 12-14 juin 2012}

rapport à la dernière crue) et le débit (figure 4): plus la crue est forte, plus le temps d'arrivée du matériel grossier (issu de l'amont) au CNPE est court.

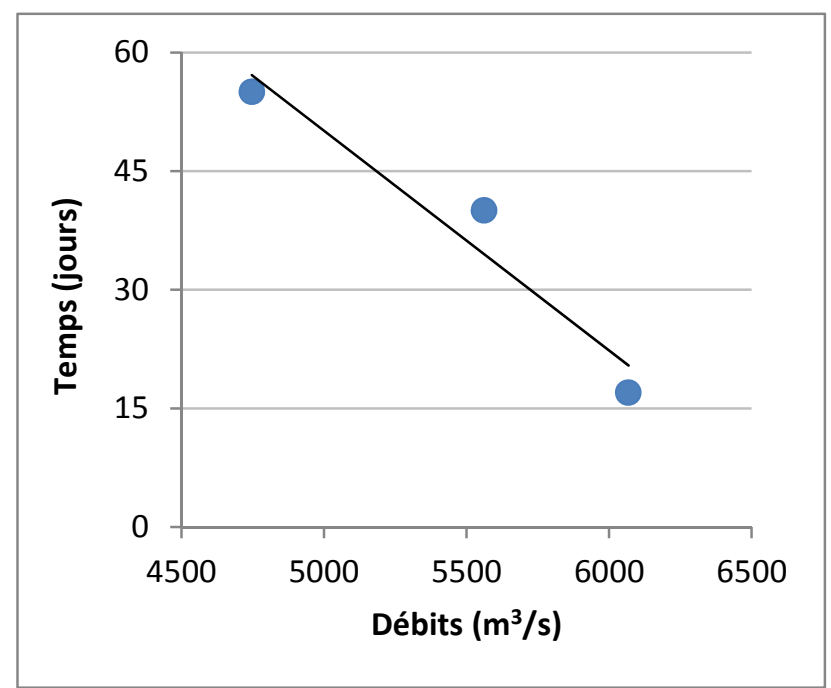

Figure 4. Décalage temporel entre crue de la Gironde et colmatage des organes de filtration (CNPE).

\section{Conclusion}

Cette étude démontre que la fraction grossière allochtone $(>315 \mu \mathrm{m})$ présente un comportement hydrodynamique influencé par les mêmes facteurs que celui des sédiments fins, mais avec son propre cycle. Pour l'estuaire de la Gironde, cette fraction grossière est alimentée par les débris végétaux provenant des bassins versants. Une fois arrivés dans l'estuaire amont, les débris végétaux connaissent des cycles de dépôts et remises en suspension, similaires à ceux des matières en suspension fines. Le temps de transit vers le secteur du CNPE apparait très dépendant des épisodes de crues.

Or le régime hydrologique de la Gironde, bien marqué par des variations saisonnières importantes, présente une diminution significative des débits au cours des 30 dernières, ce qui se répercute par un bouchon vaseux qui est de plus en plus présent en amont. Une persistance de débits faibles pourrait signifier une accumulation amont de débris végétaux dans le système bouchon vaseux - crème de vase, avec une dégradation plus ou moins marquée de ce matériel selon son temps de résidence dans l'estuaire amont. L'accumulation de débris végétaux peut être une source de nuisances pour les activités impliquant la filtration de grands volumes d'eau (pêche, filtration du circuit de refroidissement au CNPE du Blayais), du fait de transports massifs ponctuels lors des crues.

Les détritus ont été très souvent traités comme une entité statique et homogène (Odum 1963 ; WETZEL 1995). Nous avons mis en évidence que cela n'était pas le cas dans l'estuaire de la Gironde, un milieu à forte rétention de sédiment, avec d'importantes 
variations spatio-temporelles de la quantité et de la taille des débris végétaux. Il faut donc prendre en compte les mêmes paramètres (débits, marée, ..) que ceux utilisés pour le sédiment fin pour pouvoir comprendre le processus de transport des débris végétaux dans son ensemble.

\section{Références bibliographiques}

ALLEN G.P., SALOMON P., BASSOULLET P., DU PENHOAT Y., DE GRANDPRE C. (1980). Effects of tides on mixing and suspended sediment transport in macrotidal estuaries. Sedim. Geol., Vol. 26, pp 69-90. doi:10.1016/0037-0738(80)90006-8

DAVID V., TORTAJADA S., BRAHMIA A., DUPUY C., LANIESSE T., PARINET B., POUGET F., ROUSSEAU F., SIMON-BOUHET B., ROBIN F.X. (2011). Variability of salt- and freshwater marsh characteristics on the west coast of France: A spatiotemporal assessment. Water Research, Vol. 45, pp 4152-4168.

ETCHEBER H., SCHMIDT S., SOTTOLICHIO A., MANEUX E., CHABAUD G., ESCALIER J.-M., WENNEKES H., DERRIENNIC H., SCHMELTZ M., QUÉMÉNER L., REPECAUD M., WOERTHER P., CASTAING P. (2011). Monitoring water quality in estuarine environments: lessons from the MAGEST monitoring program in the Gironde fluvial-estuarine system. Hydrology and Earth System Sciences $\mathrm{n}^{\circ} 15$, pp 831-840. doi:10.5194/hess-15-831-2011

JOUANNEAU J.M., WEBER O., CREMER M., CASTAING P. (1999). Fine-grained sediment budget on the continental margin of the Bay of Biscay. Deep-Sea Research Part II: Topical Studies in Oceanography $\mathrm{n}^{\circ}$ 46, pp 2205-2220. doi:10.1016/S09670645(99)00060-0

ODUM E.P. (1963). Ecology. Holt, Rinehart and Winston, New York.

SOTTOLICHIO A., CASTAING P. (1999). A synthesis on seasonal dynamics of highly-concentrated structures in the Gironde estuary. Comptes rendus de l'Académie de sciences-série Illa: sciences de la terre et des planètes, Vol. 329, 11, pp 795-800.

SOTTOLICHIO A., CASTAING P., ETCHEBER H., MANEUX E., SCHMELTZ M., SCHMIDT S. (2011). Observations of suspended sediment dynamics in a highly turbid macrotidal estuary, derived from continuous monitoring. Journal of Coastal Research, SI 64, pp 1579-1583.

WETZEL R.G. (1995). Death, detritus, and energy-flow in aquatic ecosystems. Freswater Biol., Vol. 33, pp 83-89. doi:10.1111/j.1365-2427.1995.tb00388.x 